\title{
D’Alix à Astérix : des usages idéologiques de la bande dessinée dans la réception de l'Antiquité
}

\section{Alain Corbellari}

\section{(2) OpenEdition \\ 1 Journals}

Édition électronique

URL : http://journals.openedition.org/edl/403

DOI : $10.4000 /$ edl. 403

ISSN : 2296-5084

Éditeur

Université de Lausanne

\section{Édition imprimée}

Date de publication : 15 mai 2010

Pagination : 229-250

ISBN : 978-2-940331-22-2

ISSN : 0014-2026

Référence électronique

Alain Corbellari, «D'Alix à Astérix : des usages idéologiques de la bande dessinée dans la réception de I'Antiquité », Études de lettres [En ligne], 1-2 | 2010, mis en ligne le 15 mai 2013, consulté le 19

décembre 2020. URL : http://journals.openedition.org/edl/403 ; DOI : https://doi.org/10.4000/edl.403

(c) Études de lettres 


\section{D'ALIX À ASTÉRIX: \\ DES USAGES IDÉOLOGIQUES DE LA BANDE DESSINÉE DANS LA RÉCEPTION DE L'ANTIQUITÉ}

L'Alix de Jacques Martin et l'Astérix de Goscinny et Uderzo sont sans conteste les bandes dessinées antiquisantes les plus célèbres. La tentation est grande de les opposer terme à terme et, certes, les deux séries se prêtent à ce jeu jusqu'à un certain point. Pourtant, la fantaisie débridée d'Astérix n'est pas sans délivrer un message au fond très sérieux sur la France de la Cinquième République. A l'inverse, l'extrême documentation d'Alix reste loin de faire de cette saga un modèle d'objectivité historique et l'on s'aperçoit vite que le fantasme en déborde constamment la volonté éducative. Quelques indices montrent de plus que les auteurs des deux séries n'ont pas été totalement insensibles au message délivré par les uns et les autres. Il apparaît enfin que si la verve d'Astérix a eu tendance à s'épuiser après la mort de Goscinny, Martin a, en revanche, de son côté, évolué vers une vision plus nuancée et plus pessimiste de la Pax romana.

Dans le monde de la bande dessinée francophone, la peinture de l'Antiquité se partage diamétralement entre deux séries mythiques, illustrant chacune une modalité opposée de la peinture du passé: au grave Alix s'oppose le désopilant Astérix, aux scénarios lourdement historiques et (presque) impeccablement documentés de Jacques Martin l'univers loufoque et tout en clins d'œil de Goscinny. Mais à cette évidente opposition du sérieux et du comique se superposent d'autres différences tout aussi fondamentales qui engagent l'idéologie de nos deux séries et nous prouvent à la fois que la rigueur de la reconstitution ne garantit pas totalement l'objectivité de la peinture et que la désinvolture de la satire n'anéantit pas totalement tout référent: constat évident, sans doute, mais qui doit nous mettre en garde contre la caractérisation trop facile d'Alix en "série historique" et d'Astérix en "série parodique" comme si seul 
l'humour permettait de faire communiquer l'Antiquité romaine (ou plutôt «romano-gauloise», pour emprunter ici à Astérix aux Jeux Olympiques une expression typiquement astérixienne ${ }^{1}$ ) avec notre présent.

De fait, l'opposition de surface entre les deux séries se confirme à tant de niveaux que l'on a peine à croire que le dialogue soit entièrement dû au hasard.

Tout d'abord, il faut rappeler qu'Alix est antérieur à Astérix: le premier épisode de la série, Alix l'intrépide, date de 1948, alors que le premier Astérix, Astérix le Gaulois, est de onze ans postérieur. La rapide notoriété du héros de Jacques Martin, même si elle est sans commune mesure avec la gloire que connaîtra Astérix, n’a évidemment pu échapper à Goscinny et à Uderzo mais, à ce stade, l'hypothèse de l'influence reste encore à construire. On en trouvera cependant une preuve à notre sens décisive dans le deuxième épisode des aventures d'Alix. A bien des égards, Alix l'intrépide, qui commence par la maladroite démarcation de l'épisode initial de Ben Hur était encore un galop d'essai : si le héros y acquérait, adopté par un riche sénateur, la citoyenneté romaine qui allait fonder son statut dans le reste de la série, le récit s'attachait davantage aux péripéties, parfois extravagantes, qu'à l'idéologie de l'action. Le second tome, Le Sphinx d'or, fonde, en revanche, véritablement les poncifs de la série et ce déjà à travers la dichotomie de son action, la première partie se passant en Gaule, pays du père, la seconde en Egypte, pays de l'indéfectible ami, Enak. Surtout, la première partie commence par ce qui est aussi le récit fondateur de la saga astérixienne: la bataille d'Alésia; on y voit Vercingétorix jeter ses armes aux pieds de César (sans les écrabouiller!); on y apprend par ailleurs que le père d'Alix était un chef gaulois dont la mort a provoqué des troubles de succession dans son village. Or, quel est le nom du père d'Alix? Astorix! Cela ne s'invente pas et, quelles que soient la qualité et la pertinence du jeu de mot Astérix-astérisque, il faut, je crois, nécessairement que s'y soit superposé chez Goscinny un souvenir (inconscient?) du Sphinx d'or.

Une autre trace de l'influence d'Alix sur Astérix se lit dans la confrontation du Dieu sauvage de Martin et du Domaine des dieux de Goscinny et Uderzo: le premier raconte la brève histoire d'une colonie romaine de Cyrénaïque abandonnée prématurément suite à des troubles fomentés par des indépendantistes libyens qui introduisent dans la cité une statue aux

I. Astérix aux Jeux Olympiques, p. 23. 


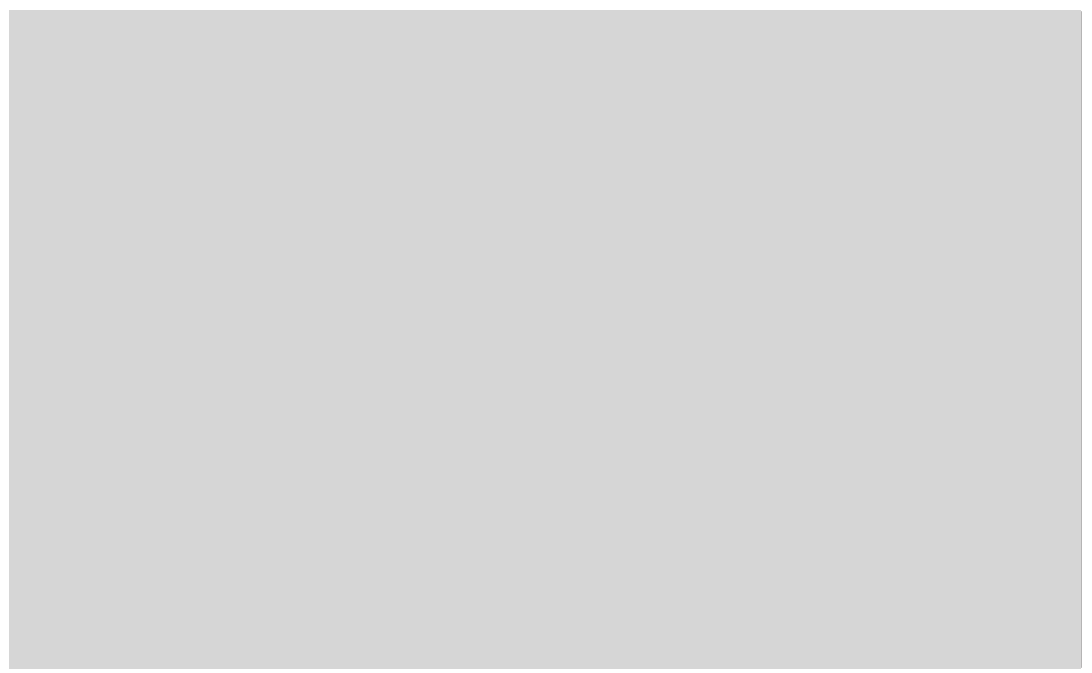

Fig. 1.

pouvoirs surnaturels, seule intervention claire de l'irrationnel - il faut le noter au passage - dans toute la saga d'Alix. L'album d'Astérix raconte quant à lui la tentative d'étouffer le village gaulois dans une ville romaine "moderne», dont il ne serait plus qu'une "amphoreville». Le Dieu sauvage commence par deux images (fig. 1) montrant le même morceau de côte cyrénaïque vu depuis l'intérieur des terres: le premier encore désert, le second faisant voir une ville déjà florissante quoique encore en construction. Qui ne songerait, en voyant ces deux illustrations, aux deux cases qui ouvrent Le Domaine des dieux (fig. 2), où le village gaulois est montré successivement entouré de forêts, puis d'immeubles romains? La seule différence est que la seconde image n'est, chez Astérix, qu'un plan-relief (on respire!). La chronologie achève de nous convaincre de l'influence: Le Dieu sauvage date de 1970, Le Domaine des dieux de 1971. Mais l'axiologie des deux récits est inversée, et Le Domaine des dieux ne peut faire figure que de réponse ironique au Dieu sauvage: si l'album d'Alix se termine mélancoliquement (en une case - fig. 3 - qui représente une dernière occurrence du paysage du début: la ville dévastée prête à retourner à l'état sauvage), par des réflexions un peu ampoulées sur la fragilité des entreprises humaines, l'Astérix s'achève au contraire sur la réaffirmation des vieilles coutumes en une manière de sacralisation de la forêt primordiale, le traditionnel banquet conclusif se déroulant pour une fois non au cœur du village mais sur les ruines $\mathrm{du}$ "domaine des dieux» 
Fig. 2.

déjà reconquises par la végétation (fig. 4). En dessinant au premier plan, appuyée à un reste de colonne, la lyre d'Assurancetourix qui, exceptionnellement (eu égard à son rôle dans l'échec du projet romain), siège au banquet, les deux auteurs se permettent même une allusion ironique au poncif romantique de la "poésie des ruines». Mais il est évident que ce symbole n'a rien, ici, de mélancolique: les exemples abondent d'ailleurs dans la série d'une assimilation non distancée de l'architecture romaine 


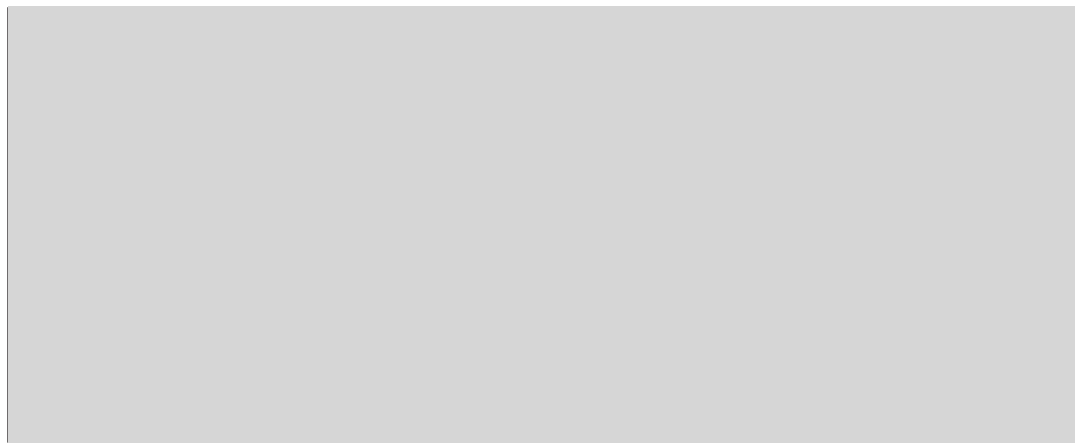

Fig. 3.

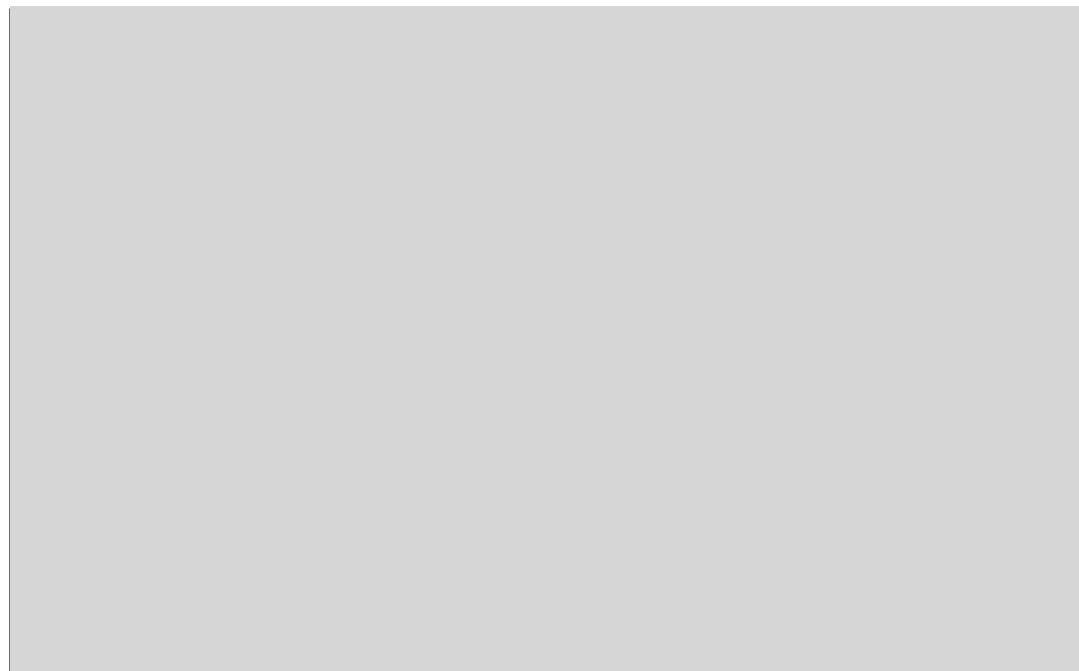

Fig. 4 .

aux plus agressives constructions des années 1960, comme dans La Serpe d'or, où se lit (p. 10, fig. 5) cette réflexion d'Astérix devant un aqueduc en travaux: "Avec leurs constructions modernes, les Romains gâchent le paysage. " Au-delà du renversement humoristique, cette position ne manque pas d'une certaine logique: pourquoi, sous prétexte que leurs aventures se passent il y a 2000 ans, les héros d'Astérix devraient-ils se priver de juger sévèrement les laideurs de "leur» modernité? On ne saurait pousser plus loin la désidéalisation de l'Antiquité; en même temps, il y a là de vraies questions qui ne sont peut-être pas aussi anachroniques 


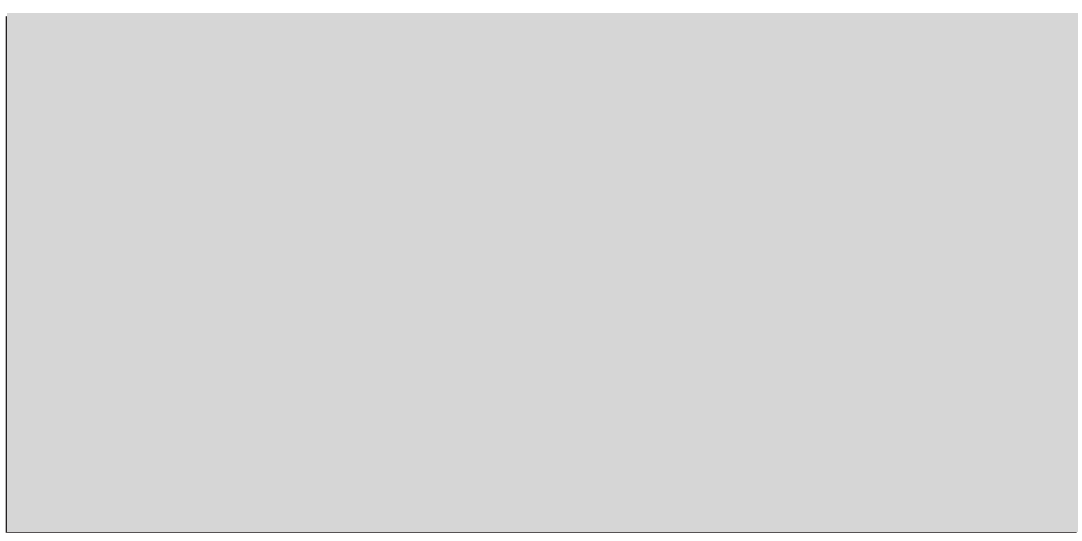

Fig. 5.

qu'elles en ont l'air, comme en témoigne a contrario l'effort quasiment désespéré de Martin pour faire taire, dans sa série, les voix qui, comme nous allons le voir, les lui suggèrent.

En dehors de ces deux exemples, cependant, on ne trouve aucune autre trace décisive de la lecture d'Alix par les auteurs d'Astérix: Goscinny et Uderzo, qui s'étaient amusés à parodier la série Barbe-Rouge de leurs amis Charlier et Hubinon, se sont abstenus de faire figurer, même à titre de clin d'œil furtif, des caricatures d'Alix ou Enak dans les aventures d'Astérix. Peur de provoquer le courroux de l'irascible Martin? Impossibilité de trouver un moyen de les rendre drôles? Cette deuxième solution en dirait long sur l'esprit de sérieux des aventures d'Alix...

On a parfois voulu faire d'Astérix un outil de propagande gaulliste et, en effet la coïncidence des dates est troublante: la même année 1959 voit le retour au pouvoir de De Gaulle et la parution du premier Astérix! Il ne faut cependant pas se laisser aller aux mirages de la ressemblance: certes, Astérix reflète fort bien l'atmosphère idéologique des débuts de la Cinquième République et l'optimisme d'une France à nouveau sûre d'elle-même, mais il y a loin jusqu'à en faire l'expression d'une adhésion pure et simple à la pensée du Général ${ }^{2}$. En réalité, Astérix milite tout au plus pour «une certaine idée de la France» ${ }^{3}$, et en 1959, quoi qu’en ait

2. Sur ces questions, voir S. Farré, «D’Aplubégalix à Alambix: les métaphores de la collaboration durant les années Astérix (1959-1977)».

3. "Toute ma vie, je me suis fait une certaine idée de la France»: première phrase des Mémoires de guerre de Charles De Gaulle (Mémoires, p. 1). 
dit le principal intéressé, cette idée n’est pas essentiellement gaulliste. Elle l'est même si peu que le plus sérieux exégète d'Astérix, dont on ne s'étonnera pas outre mesure qu'il soit allemand (!), a pu la rattacher à un imaginaire remontant, par delà des relais essentiels au XIX siècle, au moins à la Renaissance, sinon au Moyen Age ${ }^{4}$. Les remarques de cet érudit sur le fait que rien ne prédisposait les habitants anciens de l'Armorique au rôle que leur donne Astérix sont éclairantes: César compte en effet parmi les plus soumis les Gaulois de l'actuelle Bretagne, et ce n'est que dans le haut Moyen Age que naît, autour de la figure d'Arthur, le mythe de l'irréductibilité des Bretons, mythe encore bien vivace sous la Troisième République qui retournera le cliché pour faire des insoumis d'hier d'inassimilables arriérés. De fait, si l'anachronisme des menhirs, traces d'une civilisation bien antérieure à celle des Celtes, est connu de tous les lecteurs d'Astérix, la série se fonde implicitement sur une autre distorsion qui passe beaucoup plus inaperçue parce qu'elle implique l'adhésion tacite du lecteur: c'est l'idée, parfaitement controuvée, que les Bretons seraient les seuls descendants directs, sur le sol français, des anciens Gaulois. En réalité, on sait que Rome était parvenue à éradiquer complètement de la Gaule sinon toutes les coutumes (le christianisme français doit une part de son originalité au substrat celtique) du moins la langue et les institutions celtes. La Bretagne n'a été receltisée qu'au moment des Invasions et n'a donc pas plus de droit que les autres provinces de France à se réclamer de l'ancienne Gaule; «nos ancêtres les Gaulois» est, on le sait, une formule que l'on a pu seriner à tous les colonisés de la France républicaine.

J'ai tenté de montrer ailleurs ${ }^{5}$ que les habitants du village d'Astérix se répartissaient très rigoureusement dans les catégories trifonctionnelles découvertes par Georges Dumézil ( $1^{\text {re }}$ fonction, aspect diurne/Mithra: Abraracourcix, aspect nocturne/Varuna: Panoramix; $2^{\mathrm{e}}$ fonction, aspect coureur rapide/Odinnh: Astérix, aspect guerrier à la massue/Thôrr: Obélix, avec tendance prévue de ce dernier, par sa goinfrerie et son métier artisanal, à glisser vers la $3^{\mathrm{e}}$ fonction, qui comprend en outre tous les autres marchands et artisans du village; ne manquent ni l'animal compagnon du héros, Idéfix, ni la femme fatale dont tous les guerriers

4. Voir A. Stoll, Asterix: das Trivialepos Frankreichs: die Bild-und Sprachartistik eines Bestseller-Comics.

5. A. Corbellari, «Bande dessinée et trifonctionnalité». 
sont amoureux, Falbala, ni même un double parodique des personnages de $1^{\text {re }}$ fonction, Assurancetourix, dont le chant est assimilé à une magie). Hasard ou préméditation? Le fait est que la société gauloise du temps d'Astérix était bel et bien encore, grosso modo, régie par le schéma trifonctionnel indo-européen, au contraire de la société romaine de la même époque (et de fait, on ne trouve aucune trace de trifonctionnalité dans la Rome astérixienne); il n'est donc pas impossible que Goscinny ait intuitivement reproduit le schéma trifonctionnel en établissant le personnel du village gaulois.

Pour revenir au mythe de l'origine gauloise des Français, il ne saurait être question de résumer ici son histoire, même dans ses très grandes lignes. Contentons-nous de rappeler qu'il s'épanouit à la Renaissance grâce à des humanistes qui exaltent les "antiquités gauloises» face aux trop ressassées "antiquités gréco-romaines" et que, à la faveur d'une homonymie avec le verbe familier "gauler» ("plaisanter, jouer des tours»), la "gauloiserie" s'est imposée dès le XVII siècle comme une caractéristique fondamentale de l'esprit français. (Le médiéviste que je suis pourrait donc se réjouir que le mythe astérixien soit un mythe médiéval, et non antique! Mais n'insistons pas.)

Que le mythe gaulois ait été, par ailleurs, sous une forme plus rhétorique quargumentative, l'un des outils de la propagande d'un officier qui eut, entre autres bonnes fortunes, celle de s'appeler De Gaulle est également bien connu: les Mémoires de Guerre du Général ne manquent pas une occasion d'appeler les Français "Gaulois». Ici encore, il en va donc davantage de l'heureuse coïncidence que d'un dessein absolument concerté: à toutes les étapes de son élaboration, le mythe gaulois tient à l'évidence plus du «bricolage» que de la claire idéologie, la preuve en étant que le gouvernement de Vichy ne s'est pas fait faute d'en utiliser lui aussi certains éléments.

Personne, cependant, ne niera qu'il ait mieux convenu à la Résistance qu'à la Milice, et c'est bien dans cette évidence que réside l'une des clés du phénoménal succès d'Astérix. Mais est-ce suffisant pour faire de la série d'Uderzo et Goscinny une lecture unilatérale en clé gaulliste? Un problème se pose immédiatement: qui sont, dans ce cas, les Romains? Il est en effet intenable de superposer César à Hitler et les légions à la Wehrmacht. Si dans Le Tour de Gaule, ce n'est assurément pas par hasard que le réseau des partisans d'Astérix se trouve particulièrement organisé à Lyon, si telle scène de conquête (comme dans la 
deuxième image d'Astérix le Gaulois ou celle montrant le front des galères envahissant la Bretagne à la première page d'Astérix chez les Bretons) peut revêtir des allures de Blitzkrieg, les légionnaires astérixiens sont généralement plus de pauvres bidasses dépassés par les événements que de cruels envahisseurs, et César possède une dignité incompatible avec la démence hitlérienne: de tous les Romains, il est le seul à n'être (presque) jamais ridicule. Un personnage épisodique d'Astérix chez les Goths, le général Nenpeuplus, a une réplique que l'on pourrait facilement attribuer à César: «ils sont tous bêtes et je suis leur chef!» ${ }^{6}$, mais César ne se laisserait pas aller à la crise de sanglots qui secoue le bien nommé Nenpeuplus. Et il se montre (généralement) d'une grande loyauté: son pilonnage du palais de Numérobis dans Astérix et Cléopâtre est assurément une bassesse, sanctionnée comme telle par Cléopâtre elle-même, mais César agit davantage par là en "mauvais joueur " (il veut gagner son pari) qu'en tyran: aucune vie humaine n'est en jeu.

Il est au demeurant assez compréhensible que Goscinny n'ait pas voulu assombrir la joyeuse saga d'Astérix en y faisant des références trop précises aux horreurs de 39-45. Et cette retenue s'observe jusque dans sa peinture des Goths, qui caricature non tant l'Allemagne hitlérienne que celle de la Guerre de 14-18, voire même de la Guerre franco-allemande de 1870-1871: les grosses moustaches et les casques à pointes des Goths sont parfaitement bismarckiens (quant à leurs fenêtres, elles sont... gothiques!). Seule la conclusion de l'histoire, en fin de compte, peut faire référence au contexte de l'après-Hitler, car ce pays des Goths dans lequel Astérix et Panoramix ont semé les germes d'une interminable guerre civile n'est pas sans analogie avec l'Allemagne de la Guerre froide, coupée en deux pour que sa violence ne se répande plus à l'extérieur. Mais on peut tout aussi bien penser à l'Allemagne divisée d'avant Bismarck... Dans tout cela, aucune allusion au racisme (n'oublions pas que Goscinny était juif), et aucune condamnation autre de l'Allemagne que celle, toute pacifique, inspirée par une ancestrale psychologie des peuples; de fait, l'imaginaire bavarois l'emporte largement, dans la peinture astérixienne des Goths, sur la morgue prussienne.

Mais un autre élément s'oppose à l'assimilation des Romains aux Allemands: c'est la persistance de leur occupation. Certes, "nous sommes en 50 avant Jésus-Christ", mais tout concourt dans Astérix à

6. Astérix chez les Goths, p. 22. 
rendre invraisemblable le fait que la bataille d'Alésia ne serait vieille que de deux ans. Dans Le Bouclier arverne, la chronologie interne du récit exige qu'au bas mot une vingtaine d'années se soient écoulées entre la fin de la Guerre des Gaules et le présent des personnages: le temps que Coquelus ait fait fortune dans la roue - clin d'œil à l'entreprise Michelin sise à Clermont-Ferrand - ou qu'Abraracourcix, de jeune guerrier chétif qu'il était, soit devenu un chef respecté et bedonnant. De quand date l'album? De 1968. Le récit est donc parfaitement en phase avec le présent de ses premiers lecteurs, eux aussi séparés de "la dernière guerre" par une génération. Ouvrons maintenant Astérix aux Jeux Olympiques, publié en 1968 également; Agecanonix (dont c'est la première apparition) a quatrevingt-treize ans ${ }^{7}$, et s'écrie à un moment donné: "c'est parti comme en 52 !» ${ }^{8}$. Une note précise: " 52 av. J.-C.: bataille de Gergovie», mais c'est évidemment l'expression "c'est reparti comme en 14 !» qui fait sens. Agecanonix se révèle ainsi le représentant d'une catégorie de Français encore nombreuse dans les années 1960: c'est un ancien combattant de la Première Guerre mondiale! Inconséquence, incohérence? Sans doute, si on tient à conserver à la Guerre des Gaules sa valeur chronologique absolue. Mais telle n'est pas l'intention de Goscinny: le chronotope fixe, ce n'est pas celui des héros, mais celui du lecteur, et c'est à partir de lui que les allusions aux événements de l'Antiquité prendront sens. Astérix ne cesse d'être un modèle d'identification pour le Français moyen des débuts de la Cinquième République et c'est à cette aune qu'il faut juger les effets d'idéologie. Or, dans les années 60, ce ne sont évidemment plus les Allemands, mais les Américains qui font figure d'hôtes indésirables, ce qui, à vrai dire, ne contredit pas non plus l'interprétation gaulliste; de fait, tout un courant de l'opinion française peut se reconnaître sans problème dans l'idée que les Américains ont, d'une certaine manière, réussi la conquête mondiale que les Allemands ont échoué à accomplir. Le thème est banal et nous ne prétendons pas être les premiers à comparer la Pax romana à la Pax americana! Qui sait, d'ailleurs, si Astérix n'a pas joué un rôle dans la reconnaissance du parallèle?... On peut sans doute pousser la lecture gaulliste jusqu'à dire que l'ambivalence des Romains (Allemands et Américains) permet d'incarner la permanence

7. Astérix aux Jeux Olympiques, p. 27: "J'ai rajeuni - hips! - de dix ans! / Hé bien ça te fait quatre-vingt-trois ans, et tu devrais être au lit!».

8. Ibid. p. 35. 
des combats menés et des valeurs défendues par le général De Gaulle, mais ce n'est, au fond, encore une fois, que l'effet d'une coïncidence, car la prégnance des signes est toujours plus puissante que l'image de ceux qui les incarnent.

En résumé, Astérix donne l'image d'une France fière de son indépendance, méfiante envers la modernité et dont la cohésion (en dépit des bagarres!) tient en un indéfectible sens de la solidarité et de l'amitié. Il est d'ailleurs faux de faire de la bagarre un élément obligé des aventures d'Astérix: jusqu'à Astérix en Hispanie, quatorzième album de la série, seul Assurancetourix fait parfois les frais de la susceptibilité de ses concitoyens, et Panoramix voit encore, dans Le Domaine des dieux (dixseptième album!), la bagarre comme le signe d'un «plan de Jules César pour nous faire disparaître» ${ }^{9}$. Il y a donc dans la normalisation du motif de la bagarre une dérive que les auteurs de la série eux-mêmes n'ont pas vraiment su contrôler.

Revenons maintenant à Alix. Quelle est la position de ce dernier dans la Guerre des Gaules? Après être revenu dans son village et avoir soustrait son cousin Vanic à la vengeance des ennemis d'Astorix, à savoir un druide fourbe et un chef veule qui refuse de porter secours à Vercingétorix, il se fait reconnaître comme chef du village, mais observe, dans la guerre en cours, "une stricte neutralité» ${ }^{10}$ : il fait demander par une ambassade à César, qui est son ami, de laisser une colonne de ravitaillement arriver à Alésia. César refuse, tout en admirant la générosité d'Alix à qui il fait parvenir des présents. Mais un traître s'est glissé dans l'ambassade: après avoir mis hors de combat ses compagnons, il revient dire à César qu'Alix a refusé ses cadeaux. Alix est fait prisonnier par les Romains, il s'explique auprès de César qui est rassuré sur ses sentiments et lui confie alors la mission qui donne son titre au récit: le déchiffrement d'un énigme posée par la statuette d'un "sphinx d'or" et le démantèlement d'un complot antiromain basé en Egypte. Entre-temps la bataille d'Alésia a eu lieu, et Alix n'y a pas pris part! Cet escamotage bienvenu permet ainsi à l'auteur de ne pas trancher dans les sentiments qu'Alix éprouve pour ses deux patries. Il est vrai que la suite de ses aventures explicitera ses choix avec toute la précision souhaitée... Mais déjà $L e$ Sphinx d'or laisse clairement entendre où va la sympathie de l'auteur. La

9. Le Domaine des dieux, p. 36.

Io. Le Sphinx d'or, p. 10. 
Fig. 6.

société gauloise, tout d'abord, est décrite comme endémiquement faible: la disparition d'un chef fait renaître particularismes et rivalités, les tribus sont plus attachées à leur indépendance qu'à une action commune face au danger et l'état primitif des structures de vie met les Gaulois à la merci des moindres circonstances défavorables. Si les Romains ne sont pas tendres, les Gaulois sont présentés comme vindicatifs et cédant volontiers à une violence brute. L'épisode de la soumission de Vercingétorix laisse une impression ambiguë: "d'un geste violent, nous dit Martin, il jette ses armes dans la direction de César» (p. 17 - fig. 6), et le lecteur ne sait s'il doit, comme César, rendre hommage «au courage exceptionnel de cet homme» ou, comme Labiénus, s'irriter de cette morgue hors de propos. En tous les cas, l'interprétation du geste de Vercingétorix est tendancieuse, car les sources (certes romaines!) se contentent de dire que le chef vaincu "déposa» ses armes aux pieds du vainqueur (Goscinny - voir fig. 7 - se serait-il souvenu de cette lecture du geste comme un défi ?).

Quant à Astorix, il est déjà mort lorsque commencent les aventures de son fils. On nous le décrit comme un chef sage qui maintenait son village dans la paix et la prospérité. Astorix, c'est donc un âge d'or perdu, un état idéal de la société gauloise, sur lequel l'auteur n'insiste pas puisque, comme on l'a vu, son but est d'abord de souligner les faiblesses de ladite société. Un soupçon nous vient alors si l'on repense à Astérix, même s'il convient d'être prudent sur les intentions de Goscinny. Comme on l'a dit, la ressemblance des noms d'Astorix et d'Astérix prouve une influence, mais elle n'en donne pas le sens; tout se passe néanmoins comme si les auteurs d'Astérix avaient voulu, par leur hommage ambigu, souligner l'inscription de leur série dans un âge d'or de la Gaule que 


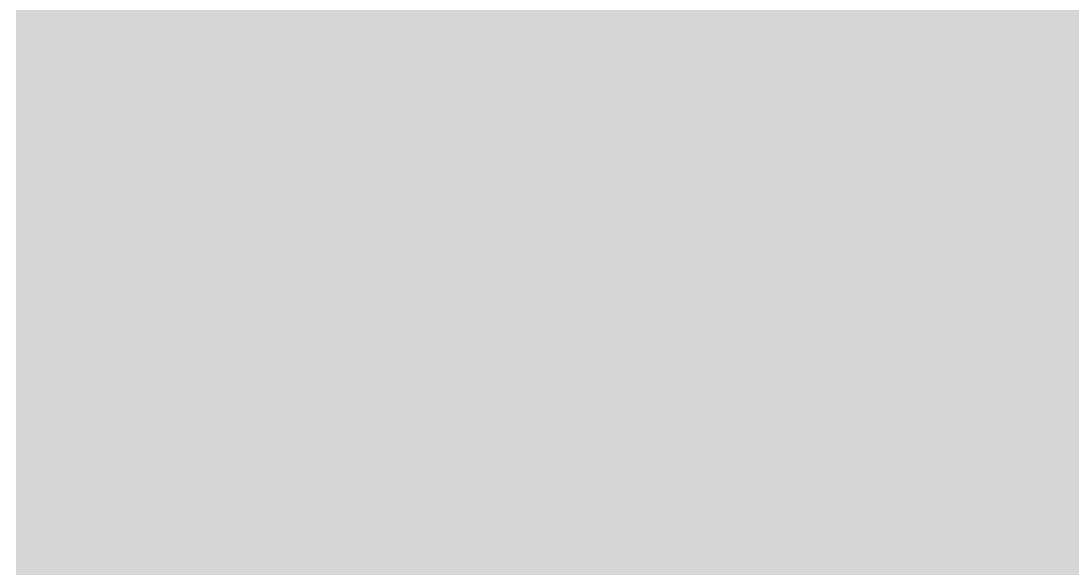

Fig. 7.

Jacques Martin considère comme révolu, si tant est qu'il ait jamais cru à son existence.

Car autant Astérix se méfie du changement, autant Alix se fait le porte-parole du grand rêve universaliste romain. Aussi toutes ses aventures - sans exception! - seront-elles bâties sur le même schéma, éminemment fantasmatique: une peuplade vaincue tente de renaître de ses cendres et de se soulever contre l'Empire, contre Rome, la cité qui a dompté l'univers et reculé, "pour le plus grand bien de l'humanité », dira-t-on, les limites de la civilisation. On pourrait même, sur le modèle narratologique de la fameuse analyse faite par Umberto Eco des aventures de James Bond ${ }^{11}$, dresser le plan d'une intrigue type des aventures d'Alix qui nous montrerait qu'il arrive toujours un moment où le héros est pris au piège et ne s'en sort qu'en déchirant progressivement sa tunique (l'analyse de ce pervers effet de strip-tease serait fort intéressante...), etc.; bref, en fin de compte ne change significativement que la nationalité des divers adversaires du héros, celui-ci ayant successivement affaire à de dangereux fanatiques gaulois (Iorix le Grand, Vercingétorix), gallo-germains (Les Légions perdues), puniques (L'Isle maudite, La Griffe noire, Le Spectre de Carthage), égyptiens (Le Sphinx d'or, Le Prince du Nil, O Alexandrie), mésopotamiens (La Tiare d'Oribal, La Tour de Babel), libyens (Le Dieu sauvage), étrusques (Le Tombeau étrusque), grecs (Le Dernier Spartiate, L'Enfant grec, Le Cheval de Troie),

II. U. Eco, «Les structures narratives chez Fleming». 
spartacistes (Le Fils de Spartacus) et même... chinois (L'Empereur de Chine), la référence romaine étant évidemment remplacée dans ce dernier exemple par celle, non moins symbolique, à l'Empire du Milieu.

Mais Jacques Martin est pris ici entre deux désirs contradictoires: celui de maintenir une chronologie extrêmement étroite, tout entière circonscrite entre la bataille d'Alésia et l'issue des luttes de César avec Pompée, donc plus étroite encore que celle d'Astérix qui s'étend au moins jusqu'à la bataille de Munda (au lendemain de laquelle se situe Astérix en Hispanie), et celui de dresser un vaste panorama du monde antique: il s'en dégage l'impression quelque peu panique d'un monde menacé sur tous ses fronts à la fois et d'une violence généralisée qui maintient son héros dans un état d'insécurité permanent, évidemment favorable à l'assimilation du chronotope alixien à la période 1939-1945. Le séminaire des sciences de l'Antiquité de l'Université de Lausanne avait, il y a quelques années, publié dans son bulletin, Chronozones, un plaisant article $^{12}$ qui tentait de redisposer dans l'ordre chronologique les aventures d'Astérix en essayant de concilier les indications internes de ses aventures avec les données de l'Histoire: le résultat, donnant sciemment à la fantaisie de Goscinny une logique qui lui était totalement étrangère, était étonnant et d'une irrésistible drôlerie, preuve par l'absurde que la décontraction astérixienne est bel et bien le fruit d'une uchronie.

Mais il n'en va pas de même chez Alix, et quelques exemples vont nous aider à préciser l'idéologie qui se dégage de ses aventures. Dans Les Légions perdues, Martin met César face au spectre terrifiant d'un retour de la barbarie en Gaule pacifiée; certes, cette invasion de la Gaule par les Germains peut évoquer le spectre de l'hégémonie nazie, mais il n'est que trop évident qu'ici l'«Empire» est valorisé. On retrouve le cousin Vanic qui présente à Alix un éloge quelque peu incongru de la "civilisation»:

Certes, les Romains sont les occupants de notre pays, mais ce sont des vainqueurs nobles et généreux qui transforment miraculeusement notre patrie. La domination romaine est bénéfique, celle des Germains serait catastrophique. Tu n'es plus retourné en Gaule depuis longtemps, Alix!... Ah! Je voudrais que tu voies certaines de nos villes à présent: c'est extraordinaire!... Elles se sont transformées d'une façon inimaginable, des maisons confortables ont remplacé nos pauvres

I2. A. Cadotte, «Astérix et l'Histoire». 
Fig. 8.

huttes et la prospérité succède à la misère!... Non, je ne veux pas que la barbarie revienne en Gaule ${ }^{13}$.

Les anachronismes et les erreurs de perspective abondent dans ce texte: comment, tout d'abord, un Gaulois aurait-il pu, au lendemain de la conquête romaine, opposer, comme un homme du $\mathrm{XX}^{\mathrm{e}}$ siècle "prospérité» et "barbarie»? Par ailleurs, on croit savoir que «Rome ne s'est pas faite en un jour» et l'invraisemblance d'un changement si rapide est encore accentué par le dessin (fig. 8) qui, en contrepoint du dithyrambe de Vanic, nous montre des scènes de la vie gallo-romaine qui n'ont pas dû se voir en Gaule avant, au bas mot, le milieu du premier siècle de notre ère. On croit d'ailleurs reconnaitre dans la villa gallo-romaine qu'il

13. Les légions perdues, p. 51. 


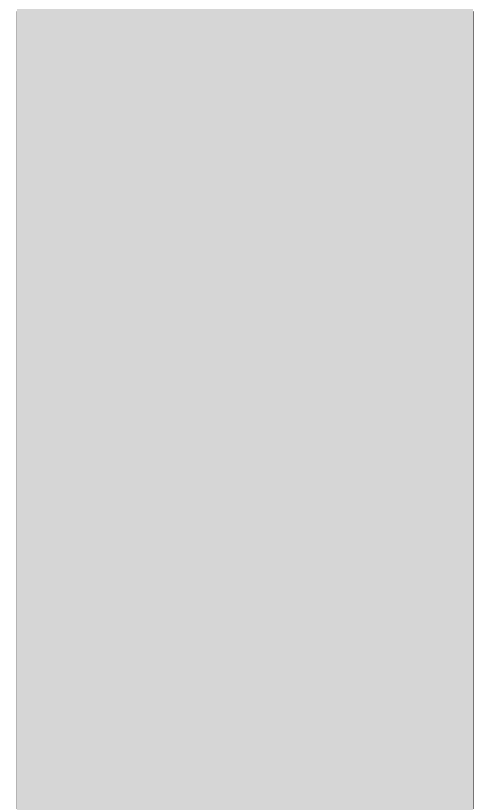

Fig. 9.

dessine la fameuse villa Kérylos, édifiée par l'archéologue Théodore Reinach au début du $\mathrm{XX}^{\mathrm{e}}$ siècle au bord de la Méditerranée, et dont la vérité archéologique n'est pas pure de tout reproche. Pire: Martin dessine aussi un curieux édifice à clocher qui ne peut être qu'une église primitive de type mérovingien, voire carolingien! ${ }^{14}$ Aussi féru qu'il soit de précision historique, Martin montre ici que, dans Alix, le message et l'impression d'ensemble priment, à l'évidence, sur la précision de la reconstitution ${ }^{15}$.

Une exposition du musée romain de Vidy, consacrée à la représentation de l'Antiquité dans la bande dessinée ${ }^{16}$, avait, au demeurant, pointé avec malice, il y a quelques années, un certain nombre d'erreurs de documentation commises par Jacques Martin: la plus cocasse était sans doute l'insertion sur l'Acropole antique d'un bâtiment en béton des années 1930. Mais le problème du rythme d'avancée de la civilisation romaine en Gaule était aussi évoqué, en particulier à propos d'une image de Iorix le Grand. Dans cet album, un mercenaire gaulois se révolte contre ses nouveaux maitres: développant une folie mégalomane à relents nietzschéens, il mourra sous les coups de ses hommes rendus à la raison. Parti de Thrace, il arrive en Gaule avec l'intention de reconquérir son pays. Le premier signe de la présence romaine est un magnifique pont en construction (fig. 9). On a eu beau jeu de montrer que non seulement un tel ouvrage d'art était en avance d'un bon siècle sur la réalité, tandis que les casques des Gaulois dataient sans

I4. Je remercie pour ces remarques mon collègue Michel Fuchs, dont l'œil exercé a vivement réagi aux images que j'ai montrées lors de ma conférence.

I5. Pour une bonne introduction à l'univers d'Alix et à ce qu'en dit son créateur, voir Th. Groensteen, Avec Alix: l'univers de Jacques Martin.

16. Anti-Comix. L'Antiquité dans la bande dessinée, exposition de la Skulpturhalle de Bâle, présentée au musée romain de Lausanne-Vidy du 22 septembre 2000 au 14 janvier 2001. 
doute, quant à eux, de l'époque de Brennus: cette image concaténait par conséquent des réalités distantes d'un demi-millénaire! Mais l'épisode du pont a une valeur démonstrative dont Martin ne pouvait se passer: répondant (cette fois-ci sans doute de manière parfaitement fortuite) à la remarque d'Astérix qui trouvait que les constructions romaines "gâchaient le paysage" (fig. 5), Iorix n'a de cesse d'avoir détruit ce symbole de l'oppression. Mais alors que l'opinion d'Astérix était destinée à rencontrer la sympathie du lecteur, il n'est que trop évident qu'ici Iorix n'est qu'un épouvantable barbare qui ne comprend rien non seulement à la civilisation, mais pire encore, à la beauté. Après tout, les cinq cents ans de distance entre son équipement et la construction romaine ne sont pas aussi absurdes qu'on pourrait le croire sur un plan symbolique: Iorix est bel et bien, dans ce récit, un homme d'un autre temps!

Dans Vercingétorix, cependant, nous voyons se fissurer cette éthique de la collaboration. Dans cet album tardif, c'est le grand chef celte luimême (en fin de série, Martin se fait moins exigeant envers la vraisemblance historique) qui, s'évadant grâce à Pompée, lequel cherche par là à nuire à son vieil ennemi César, organise sa vengeance avant d'être in extremis occis sous les yeux du conquérant des Gaules. Au début de l'histoire, Vercingétorix (représenté en rouquin encore jeune, fig. 10, sans la moindre ressemblance avec le moustachu à cheveux noirs du Sphinx d'or, voir fig. 6) lance à la tête d'Alix, qui l'évite de justesse, un pot de fleurs, et le harangue vertement:

Traitre! Alors que tant de guerriers sont morts pour chasser les envahisseurs, toi, un Gaulois, tu mangeais le pain de Rome ${ }^{17}$.

17. Vercingétorix, p. 6. 
Ne laissant pas à Alix le temps de réagir, c'est Pompée lui-même (excusez du peu!), qui répond au chef gaulois, en lui adressant ces propos lénifiants et quelque peu sophistiques:

Non! Tu fais erreur. Alix est le fils d'Honorus Gracchus Galla, noble citoyen romain qui occupa de glorieuses fonctions dont la République le chargea. Ce jeune homme s'appelle d'ailleurs Alix Gracchus. Il a des origines étrangères mais beaucoup de Romains sont dans ce cas: entre les Italiotes, les Etrusques, les Grecs, les Cisalpins et d'autres peuples, les citoyens de l'Urbs sont un peu noyés ${ }^{18}$.

Difficile ici encore de ne pas sourire en lisant cette réplique qui témoigne d'une perception de la société romaine qui sent davantage l'historien moderne que le général de la République. Le message est clair: Rome, c'est la société multiculturelle de l'avenir, dans laquelle les particularismes sont destinés à s'effacer pour le plus grand profit d'une civilisation universelle. Mais, outre qu'ici la lecture en clé américaine semble plus adéquate que la lecture en clé nazie, il faut remarquer qu'Alix est resté muet: il nous est donc impossible de savoir s'il approuve l'analyse de Pompée, et la suite du récit va nous montrer que tel n'est sans doute pas tout à fait le cas. De fait, le tour de passe-passe par lequel la gallicité d'Alix a été évacuée ne fait pas totalement oublier l'accusation de "trầtrise" de Vercingétorix, et ce d'autant plus qu'un autre personnage récurrent de la série pourrait lui adresser le même reproche: Alix n'était-il pas jusque là un ami de César? Pompée le lui rappelle d'ailleurs, se félicitant qu’il ait «changé de camp». Ce à quoi Alix rétorque:

Non, il se trouvait que César défendait à ce moment-là le parti du juste. Cette fois c'est toi mais je suis certain que cette affaire sert parfaitement tes intérêts ${ }^{19}$.

Il s'avère donc, en définitive, peut-être un peu trop simple d'opposer Astérix résistant à Alix collabo. L'ambivalence de la peinture des Romains, tantôt assimilés aux Allemands, tantôt aux Américains, est en fait commune aux deux séries et, si elle permet d'opposer efficacement Astérix à toutes les bêtes noires du gaullisme, elle rend plus ambiguë la position réelle d'Alix dans ce monde auquel il commence par s'identifier,

I8. Ibid. p. 6.

I9. Ibid. p. 7. 
mais dont il semble progressivement se détacher dans les derniers albums de la série. Qu'il y ait chez le dessinateur d'Alix une pente qui l'aurait sans doute fait acquiescer jusqu' à un certain point, en 1940, au "nouvel ordre" que d'aucuns voulaient imposer à l'Europe, cela semble certes possible. Ce qui le sauve, cependant, c'est cette part d'idéalisme chevaleresque que conserve son héros, lequel s'insurge constamment contre la violence qui l'entoure, et est même plus d'une fois tenté de prendre le parti des révoltés et de leur aspiration à un monde plus calme, vierge de toute ingérence extérieure. Mais "tenté» est bien le mot, la sympathie d'Alix, dans ces cas étant généralement liée à de jolies jeunes femmes dont plus d'une s'avère en fin de compte une exaltée, voire une dévoratrice. Alix, d'ailleurs, leur résiste toujours et n'éprouve jamais beaucoup plus qu'une sympathie attendrie pour ces créatures qui ne sauraient supplanter, dans son affection, son compagnon Enak (voir Le dernier Spartiate, Le Dieu sauvage, Le Prince du Nil, etc.) ; le choix est même plus d'une fois explicité: il n'est en effet pas rare qu'Alix renonce à la compagnie d'une femme pour partir à la recherche de son ami prisonnier ou disparu (voir Le Spectre de Carthage). Des femmes meurent pour lui: il n'a pour elles qu'une compassion navrée. Mais en quoi ces sympathies sporadiques d'Alix concernent-elles l'idéologie de la série? En cela qu'elles révèlent chez le héros une composante sentimentale et idéaliste qui le sauve des acquiescements trop faciles à la Realpolitik de Rome. Dans Vercingétorix, il se brouille même avec César et évolue, dès lors, de plus en plus vers un personnage de paria et d'aventurier sans patrie. Déjà, on l'a vu, le constat pessimiste du Dieu sauvage dénonçait la vanité de toute entreprise civilisatrice.

Oserait-on dire qu'il sera beaucoup pardonné à Jacques Martin, parce qu'il aura beaucoup douté?

Alain Corbellari

Université de Lausanne 


\section{BIBLIOGRAPHIE}

Cadotte, Alain, «Astérix et l'Histoire», Chronozones, 6 (2000), p. 46-55. Corbellari, Alain, "Bande dessinée et trifonctionnalité», Belphégor, IV/1 (novembre 2004) http://etc.dal.ca/belphegor/vol4 nol/ articles/0401_Corbel trifon fr.html.

Eco, Umberto, "Les structures narratives chez Fleming", traduit de l'italien par Myriem Bouzaher, in De Superman au surhomme, Paris, Grasset, 1993, p. 163-207.

FARré, Sébastien, "D’Aplusbégalix à Alambix: les métaphores de la collaboration durant les années Astérix (1959-1977)», in Objectif bulles, éd. Michel Porret, Genève, Georg, "L'Equinoxe», p. 181-202.

Gaulle, Charles de, Mémoires, Paris, Gallimard, "Bibliothèque de la Pléiade», 2000.

Groensteen, Thierry, Avec Alix: l'univers de Jacques Martin, édition revue et augmentée, Tournai, Casterman, 2002.

Stoll, André, Asterix: das Trivialepos Frankreichs: die Bild-und Sprachartistik eines Bestseller-Comics, Köln, DuMont, 1977; version française revue et augmentée, traduite par l'auteur et révisée par Alain Morot: Astérix: l'épopée burlesque de la France, Bruxelles, Editions Complexe, 1978. 
Références des illustrations

Fig. 1 et 3:

Jacques Martin, Alix. Le Dieu sauvage, Tournai, Casterman, 1970, p. 3 cases 1 et 2 et p. 56 dernière case.

Fig. 2 et 4 :

René Goscinny, Albert Uderzo, Astérix. Le Domaine des dieux, Paris Dargaud, 1971, p. 4 cases 1 et 2 et p. 47, dernière case.

Fig. 5:

René Goscinny, Albert Uderzo, Astérix. La Serpe d'or, Paris, Dargaud, 1962, p. 10 case 4 .

Fig. 6:

Jacques Martin, Alix. Le Sphinx d'or, Tournai, Casterman, 1971, p. 17 case 11 .

Fig. 7:

René Goscinny, Albert Uderzo, Astérix. Le Bouclier arverne, Paris, Dargaud, 1968, p. 5 case 1.

Fig. 8:

Jacques Martin, Alix. Les Légions perdues, Tournai, Casterman, 1965, p. 51 cases 5 et 6 (au centre).

Fig. 9 :

Jacques Martin, Alix. Iorix le grand, Tournai, Casterman, 1972, p. 43 case 4 .

Fig. 10:

Jacques Martin, Alix. Vercingétorix, Tournai, Casterman, 1985, p. 6 case 1 . 
